\title{
A NEW EXPLICIT FORMULA FOR BERNOULLI NUMBERS INVOLVING THE EULER NUMBER
}

\section{A PREPRINT}

\section{Sumit Kumar Jha}

International Institute of Information Technology

Hyderabad, India

kumarjha.sumit@research.iiit.ac.in

June 13, 2019

\begin{abstract}
In this brief note, we derive a new explicit formula for Bernoulli numbers in terms of the Stirling numbers of the second kind and the Euler numbers. As a corollary of our result, we obtain an explicit formula for the Euler numbers in terms of the Stirling numbers of the second kind.
\end{abstract}

Keywords Bernoulli numbers · Stirling numbers of the second kind · Euler numbers · Polylogarithm function AMS Classification: 11B68

There are many known explicit formulas known for the Bernoulli Numbers [1]. We prove the following.

Theorem 1. We have

$$
B_{r+1}=-\frac{r+1}{4\left(1+2^{-(r+1)}\left(1-2^{-r}\right)\right)}\left(\sum_{k=1}^{r}(-1)^{k} \cdot \frac{S(r, k)}{k+1} \cdot\left(\frac{3}{4}\right)^{(k)}+4^{-r} E_{r}\right),
$$

where $S(r, k)$ denotes the Stirling numbers of the second kind, $x^{(n)}=(x)(x+1) \cdots(x+n-1)$ denotes the rising factorial, and $E_{r}$ denotes the Euler number.

Proof. We begin with the following result

$$
\frac{\sin n \pi}{\pi} \int_{0}^{\infty} x^{n-1} \frac{\mathbf{L} \mathbf{i}_{s}(-x)}{1+x} d x=\zeta(s)-\zeta(s, 1-n),
$$

where $\mathbf{L} \mathbf{i}_{s}(-x)$ denotes the Polylogarithm function, $\zeta(s)$ is the Riemann zeta function, and $\zeta(s, 1-n)$ is the Hurwitz zeta function. The above integral is valid for all $s \in \mathbb{C} \backslash\{1\}$, and $n \in \mathbb{R}$. This integral can be obtained from formula 3.2.1.6 in the book [2].

Plugging $n=3 / 4$ in the above, and $s=-r$, a negative integer, we get

$$
\int_{0}^{\infty} x^{-1 / 4} \frac{\mathbf{L i}_{-r}(-x)}{1+x} d x=\sqrt{2} \pi\left(\frac{B_{r+1}(1 / 4)-B_{r+1}}{r+1}\right) .
$$

Now, we use the following representation from the note [3]

$$
\mathbf{L i}_{-r}(-x)=\sum_{k=1}^{r} k ! S(r, k)\left(\frac{1}{1+x}\right)^{k+1}(-x)^{k},
$$

which can be easily proved using induction on $r$. 
As a result, we have

$$
\begin{aligned}
& \int_{0}^{\infty} x^{-1 / 4} \frac{\mathbf{L i}-r}{1+x} d x=\sum_{k=1}^{r}(-1)^{k} \cdot k ! S(r, k) \int_{0}^{\infty} \frac{x^{k-1 / 4}}{(1+x)^{k+2}} d x \\
&= \sum_{k=1}^{r}(-1)^{k} \cdot k ! S(r, k) \cdot \frac{\Gamma(k+3 / 4) \Gamma(5 / 4)}{\Gamma(k+2)} \\
&=\sum_{k=1}^{r}(-1)^{k} \cdot \frac{S(r, k)}{k+1} \cdot \Gamma(k+3 / 4) \Gamma(5 / 4) \\
&=\sum_{k=1}^{r}(-1)^{k} \cdot \frac{S(r, k)}{k+1} \cdot\left(\frac{3}{4}\right) \cdot\left(\frac{3}{4}+1\right) \cdots\left(\frac{3}{4}+k-1\right) \Gamma(3 / 4) \Gamma(5 / 4) \\
&=\sum_{k=1}^{r}(-1)^{k} \cdot \frac{S(r, k)}{k+1} \cdot\left(\frac{3}{4}\right) \cdot\left(\frac{3}{4}+1\right) \cdots\left(\frac{3}{4}+k-1\right) \frac{\pi}{2 \sqrt{2}} \\
&=\sum_{k=1}^{r}(-1)^{k} \cdot \frac{S(r, k)}{k+1} \cdot\left(\frac{3}{4}\right)^{(k)} \frac{\pi}{2 \sqrt{2}},
\end{aligned}
$$

here $\Gamma(\cdot)$ is the Gamma function.

But, from [4], we have

$$
\frac{B_{r+1}(1 / 4)-B_{r+1}}{r+1}=\frac{\left(-2^{-(r+1)}\left(1-2^{-r}\right) B_{r+1}-4^{-(r+1)}(r+1) E_{r}-B_{r+1}\right)}{r+1} .
$$

Thus, we have

$$
B_{r+1}=-\frac{r+1}{4\left(1+2^{-(r+1)}\left(1-2^{-r}\right)\right)}\left(\sum_{k=1}^{r}(-1)^{k} \cdot \frac{S(r, k)}{k+1} \cdot\left(\frac{3}{4}\right)^{(k)}+4^{-r} E_{r}\right) .
$$

If we let $r=2 l$, an even integer, in equation (1) we immediately obtain

\section{Corollary 1.}

$$
E_{2 l}=-4^{2 l} \sum_{k=1}^{2 l}(-1)^{k} \cdot \frac{S(2 l, k)}{k+1} \cdot\left(\frac{3}{4}\right)^{(k)} .
$$

Remark 1. The cases $n=1 / 2$ and $n \rightarrow 1$ of equation (2) have been discussed in the note [5].

\section{References}

1 Gould, Henry W. "Explicit formulas for Bernoulli numbers." The American Mathematical Monthly 79.1 (1972): 44-51.

2. Handbook of Mellin Transforms by Yu. Brychkov, O. Marichev, N, Savischenko, 2019.

3. Stirling Numbers and Polylogarithms by Steven E. Landsburg. Link: http://www.landsburg.com/query.pdf

4. Weisstein, Eric W. "Bernoulli Polynomial." From MathWorld-A Wolfram Web Resource.

5. Jha, S.K., 2019. Two new explicit formulas for the Bernoulli Numbers. arXiv preprint arXiv:1905.11216. 